\title{
Can Magnetic Monopoles and Massive Photons Coexist in the Framework of the Same Classical Theory?
}

\author{
C. Cafaro, ${ }^{1}$ S. Capozziello, ${ }^{2,3}$ Ch. Corda, ${ }^{4,5}$ and S. A. Ali ${ }^{1}$ \\ ${ }^{1}$ Department of Physics, University at Albany-SUNY, 1400 Washington Avenue, Albany, \\ NY 12222, USA \\ ${ }^{2}$ Dipartimento di Scienze Fisiche, Università di Napoli "Federico II," Via Cinthia, 80126 Napoli, Italy \\ ${ }^{3}$ INFN Sezione di Napoli, Complesso Universitarion di Monte Sant' Angelo, Via Cinthia, \\ 80126 Napoli, Italy \\ ${ }^{4}$ INFN Sezione di Pisa and Università di Pisa, Via F. Buonarroti 2, 56127 Pisa, Italy \\ ${ }^{5}$ European Gravitational Observatory (EGO), Via E. Amaldi, 56021 Cascina (PI), Italy
}

Correspondence should be addressed to S. Capozziello, capozziello@na.infn.it

Received 5 September 2007; Accepted 2 November 2007

Recommended by Joseph Formaggio

It is well known that one cannot construct a self-consistent quantum field theory describing the nonrelativistic electromagnetic interaction mediated by massive photons between a point-like electric charge and a magnetic monopole. We show that, indeed, this inconsistency arises in the classical theory itself. No semiclassic approximation or limiting procedure for $\hbar \rightarrow 0$ is used. As a result, the string attached to the monopole emerges as visible also if finite-range electromagnetic interactions are considered in classical framework.

Copyright (c) 2007 C. Cafaro et al. This is an open access article distributed under the Creative Commons Attribution License, which permits unrestricted use, distribution, and reproduction in any medium, provided the original work is properly cited.

In his classical works, Dirac showed that the existence of a magnetic monopole would explain the electric charge quantization [1,2]. This is known as the Dirac quantization rule. There exist various arguments based on quantum mechanics, theory of representations, topology, and differential geometry on behalf of Dirac rule $[3,4]$. Dirac formulation of magnetic monopoles takes into account a singular vector potential. Other approaches exist where two nonsingular vector potentials, related through a gauge transformation, are used [5, 6]. Finite-range electrodynamics is a theory with nonzero photon mass. It is an extension of the standard theory and is fully compatible with experiments. The existence of Dirac monopole in massless electrodynamics is compatible with the above quantization condition if the string attached to the monopole is invisible. The quantization condition can be obtained either with the help of gauge invariance 
or angular momentum quantization. In massive electrodynamics, both these approaches are no longer applicable [7]. These conclusions are formulated in a quantum framework which is a quantized version of the classical one. The Hamiltonian formulation and the problems involved in quantization of Dirac theory of monopoles have been extensively discussed in the past and are still an active field of research [8,9]. Major work on the quantum field theory of magnetic charges has been developed by Schwinger [10-12] and Zwanziger [13]. Recent work on constructing a satisfactory classical relativistic framework for massive electrodynamics and magnetic monopoles from a geometrical point of view has been considered in [14, 15]. A complete update on the experimental and theoretical status of monopoles is presented in [16].

In this paper, we consider the problem of constructing the static limit of a consistent classical, nonrelativistic electromagnetic theory describing a point-like electric particle with charge $e$ and mass $m$ moving in the field of a fixed composite monopole of charge $e_{m}$, where their mutual interaction is mediated by massive carrier gauge fields. The total magnetic field $\vec{B}$ is comprised of point-like magnetic charge, a semi-infinite string along the negative $z$-axis and diffuse magnetic field contributions. We impose that the electrically charged particle must never pass through the string (Dirac-veto) [17] and therefore the motion of the test charged particle is constrained to region of motion $R^{+}:=\left\{(r, \theta, \varphi): r \in \mathbb{R}_{0}^{+}, \theta \in[0, \pi), \varphi \in[0,2 \pi]\right\}$. It is known that no spherically symmetric diffuse magnetic field solutions are allowed in Maxwell classical electrodynamics with massive photons and magnetic monopoles [7]. Requiring the theory presented here is endowed with a well-defined canonical Poisson bracket structure, it is shown that the total angular momentum is the generator of rotations. Furthermore, by demanding proper transformation rules under spatial rotations for the allowed magnetic vector field solutions, it is shown that only spherically symmetric diffuse magnetic fields satisfy the Lie algebra of the system. This leads to conclude that the permitted solutions to the generalized Maxwell theory are incompatible with the Lie algebra of the Hamiltonian formulation. As a consequence, any quantization procedure applied to this classical theory would lead to an inconsistent quantum counterpart.

Maxwell equations with nonzero photon mass and magnetic charge follow from a standard variational calculus [18-20] of the Maxwell-Proca-Monopole action functional. The field equations for the electromagnetic 4-vector potential $A_{\mu}$, together with the Bianchi identities and Lorenz gauge condition $\partial_{\mu} A^{\mu}=0$, lead to the generalized Maxwell equations in three dimensions as follows:

$$
\begin{gathered}
\vec{\nabla} \cdot \vec{E}=4 \pi \rho_{e}-m_{r}^{2} A_{0}, \quad \vec{\nabla} \times \vec{E}=-c^{-1} \partial_{t} \vec{B}-4 \pi c^{-1} \vec{j}_{m}, \\
\vec{\nabla} \cdot \vec{B}=4 \pi \rho_{m^{\prime}} \quad \vec{\nabla} \times \vec{B}=4 \pi c^{-1} \vec{j}_{e}+c^{-1} \partial_{t} \vec{E}-m_{r}^{2} \vec{A},
\end{gathered}
$$

where $m_{\gamma}=\omega / c$ and $\omega$ is the frequency of the photon. In absence of electric fields, charges, and currents, as well as the absence of magnetic current, the static monopole-like solution of this system is

$$
\vec{B}=\vec{B}^{(\text {Dirac })}+\vec{B}_{\gamma}
$$

where $\vec{B}^{(\text {Dirac) }}$ is the standard Dirac magnetic field

$$
\vec{B}^{\text {(Dirac) }}=\frac{e_{m}}{r^{2}} \widehat{r}
$$


whose divergence and curl are given by

$$
\vec{\nabla} \cdot \vec{B}^{(\text {Dirac })}=4 \pi e_{m} \delta^{(3)}(\vec{r}), \quad \vec{\nabla} \times \vec{B}^{(\text {Dirac })}=0 .
$$

The diffuse magnetic field $\vec{B}_{\gamma}(\vec{r})$ is given by the following general expression

$$
\vec{B}_{\gamma}(\vec{r})=b_{\gamma}^{(1)}(r, \widehat{n} \cdot \vec{r}) \vec{r}+b_{\gamma}^{(2)}(r, \widehat{n} \cdot \vec{r}) \widehat{n}
$$

where $b_{\gamma}^{(1)}$ and $b_{\gamma}^{(2)}$ are general scalar field functions, and $\widehat{n}$ is a unitary vector along the monopole string. The magnetic field $\vec{B}_{\gamma}(\vec{r})$ is such that

$$
\vec{\nabla} \cdot \vec{B}_{\gamma}=0, \quad \vec{\nabla} \times \vec{B}_{\gamma}=-m_{\gamma}^{2}\left(\vec{A}^{(\text {Dirac })}+\vec{A}_{\gamma}\right) .
$$

The vector $\vec{A}^{\text {(Dirac) }}$ is the standard singular vector potential representing the field of a fixed monopole;

$$
\vec{A}^{(\text {Dirac })}(\vec{r})=\frac{e_{m}}{r^{2}} \frac{\sin (\theta)}{1+\cos (\theta)}(\widehat{n} \times \vec{r}), \quad \theta \neq \pi
$$

with semi-infinite singularity line oriented along the negative $z$-axis, where $e_{m}$ is the magnetic charge. The vector potential $\vec{A}_{\gamma}(\vec{r})$ is given by the following general expression

$$
\vec{A}_{\gamma}(\vec{r})=e_{m} m_{\gamma}^{2} f_{\gamma}\left(m_{\gamma} r, m_{\gamma} \vec{r} \cdot \widehat{n}\right)(\widehat{n} \times \vec{r}),
$$

where $f_{\gamma}$ is a generic scalar field function. Because of the second equation in (6), it is clear that no spherically symmetric diffuse magnetic field solutions are allowed, that is to say, solutions like

$$
\vec{B}_{\gamma}(\vec{r})=B_{\gamma}(r) \widehat{r}
$$

are not allowed.

On the other hand, it is known that the classical nonrelativistic theory describing the massless electromagnetic scattering of an electric charge from a fixed magnetic monopole does have a Hamiltonian formulation [21]. With this result in mind, let us consider the classical nonrelativistic theory describing a point-like electric particle with charge $e$ and mass $m$ moving in the field of a fixed monopole of charge $e_{m}$, but let us suppose that the electromagnetic interaction is mediated by massive photons. The total magnetic field $\vec{B}$ is comprised of the point-like magnetic charge, string, and diffuse magnetic field contributions as follows:

$$
\begin{aligned}
\vec{B} & =\vec{B}^{\text {(Dirac) }}+\vec{B}_{\gamma} \\
& =\left[\vec{\nabla} \times \vec{A}^{(\text {Dirac })}+e_{m} \vec{f}(\vec{r})\right]+\vec{\nabla} \times \vec{A}_{\gamma} \\
& =\vec{\nabla} \times \vec{A}+e_{m} \vec{f}(\vec{r}), \\
\vec{A} & =\vec{A}^{\text {(Dirac) }}+\vec{A}_{\gamma},
\end{aligned}
$$

where

$$
\|\vec{f}(\vec{r})\|=4 \pi \delta(x) \delta(y) \Theta(-z)=\frac{4 \pi}{r^{2}} \frac{\delta(\theta) \delta(\varphi)}{\sin \theta} \Theta(-\cos \theta)
$$


is the string function having support only along the line $\widehat{n}=-\widehat{z}$ and passing through the origin while $\Theta$ is the Heaviside step function.

The classical Newtonian equation of motion describing this system is

$$
m \frac{d^{2} \vec{r}}{d t^{2}}-\frac{e}{c} \frac{d \vec{r}}{d t} \times(\vec{\nabla} \times \vec{A})-\frac{e e_{m}}{c} \frac{d \vec{r}}{d t} \times \vec{f}(\vec{r})=0 .
$$

The Hamiltonian that gives rise to the above equations of motion reads

$$
H_{\text {total }}(\vec{p}, \vec{r})=\frac{(\vec{p}-(e / c) \vec{A})^{2}}{2 m}+H_{\text {string, }} \quad H_{\text {string }}=-\frac{e e_{m}}{c} \int\left(\frac{d \vec{r}}{d t} \times \vec{f}(\vec{r})\right) \cdot d \vec{r}
$$

We impose that the electrically charged particle must never pass through the string (Diracveto) and therefore the classical equation of motion in the allowed region of motion $R^{+}:=$ $\left\{(r, \theta, \varphi): r \in \mathbb{R}_{0}^{+}, \theta \in[0, \pi), \varphi \in[0,2 \pi]\right\}$ is given by

$$
m \frac{d^{2} \vec{r}}{d t^{2}}-\frac{e}{c} \frac{d \vec{r}}{d t} \times(\vec{\nabla} \times \vec{A})=0
$$

The restricted Hamiltonian associated with (14) is given by

$$
\begin{aligned}
H(\vec{p}, \vec{r}) & =\frac{(\vec{p}-(e / c) \vec{A})^{2}}{2 m}=\frac{(\vec{P} \cdot \widehat{r})^{2}}{2 m}+\frac{(\vec{P} \cdot \widehat{\theta})^{2}}{2 m} \\
& =\frac{(\vec{P} \cdot \widehat{r})^{2}}{2 m}+\frac{\vec{L}^{2}}{2 m r^{2}}=\frac{(\vec{P} \cdot \widehat{r})^{2}}{2 m}+\frac{\left(\vec{J}^{2}-\vec{s}^{2}\right)}{2 m r^{2}},
\end{aligned}
$$

where $\vec{p}=m(d \vec{r} / d t)+(e / c) \vec{A}$ is the canonical momentum vector, $\vec{P}=\vec{p}-(e / c) \vec{A}=m(d \vec{r} / d t)$ is the kinetic momentum vector, $\vec{L}=\vec{r} \times \vec{P}$ is the orbital angular momentum of the system, and $\vec{J}=\vec{L}+\vec{s}$ is the total angular momentum such that $\vec{J} \cdot \vec{s}=0$, where

$$
\begin{aligned}
\vec{s} & =(4 \pi c)^{-1} \int[\vec{r} \times(\vec{E} \times \vec{B})] d^{3} \vec{r} \\
& =\vec{s}_{\text {massless }}+\frac{e}{4 \pi c} \int d \vec{r} \vec{r} \times\left[\frac{\vec{r}}{r^{3}} \times \vec{B}_{\gamma}(\vec{r}-\vec{R})\right]
\end{aligned}
$$

with $\vec{s}_{\text {massless }}=\left(e e_{m} / c\right) \widehat{R}[21-23]$, and $\vec{R}$ is the relative vector position between the monopole and the electric charge. The vector $\vec{s}$ is taken as an angular momentum with independent degrees of freedom and must obey the following classical Poisson-bracket relation

$$
\left\{s_{i}, s_{j}\right\}=-\varepsilon_{i j k} s_{k} .
$$

Observe that $H_{\text {total }}(\vec{p}, \vec{r})$ is not spherically symmetric due to the occurrence of $H_{\text {string }}$ and even in the restricted case of $H(\vec{p}, \vec{r})$, the term $\vec{\nabla} \times \vec{A}_{\gamma}$ breaks rotational invariance since

$$
\begin{aligned}
\frac{d \vec{r}}{d t} \times\left(\vec{\nabla} \times \vec{A}_{\gamma}\right) & =\frac{d \vec{r}}{d t}\left(\vec{\nabla} \cdot \vec{A}_{\gamma}\right)-\left(\frac{d \vec{r}}{d t} \cdot \vec{\nabla}\right) \vec{A}_{\gamma} \\
& =-\left(\frac{d \vec{r}}{d t} \cdot \vec{\nabla}\right) \vec{A}_{\gamma} \neq 0 \text { in general } .
\end{aligned}
$$


We made use of the transversality condition $\vec{\nabla} \cdot \vec{A}_{\gamma}=0$ in computing $(d \vec{r} / d t) \times\left(\vec{\nabla} \times \vec{A}_{\gamma}\right)$ [7] . Furthermore, we emphasize that we may obtain a spherically symmetric Hamiltonian, provided the auxiliary condition $(d \vec{r} / d t)_{k} \partial_{k}\left(A_{\gamma}\right)_{j}=0$ for all $j=1,2,3$ is satisfied. Such condition is however unnecessary for our present analysis.

The Poisson brackets between two generic functions $f(\vec{p}, \vec{r}, t)$ and $g(\vec{p}, \vec{r}, t)$ of the dynamical variables $\vec{p}$ and $\vec{r}$ are defined as

$$
\{f(\vec{p}, \vec{r}, t), g(\vec{p}, \vec{r}, t)\} \stackrel{\text { def }}{=} \sum_{i}\left(\partial_{p_{i}} f \partial_{r_{i}} g-\partial_{r_{i}} f \partial_{p_{i}} g\right),
$$

and the basic canonical Poisson bracket structure for the conjugate variables is given by

$$
\left\{r_{i}, r_{j}\right\}=0, \quad\left\{r_{i}, p_{j}\right\}=-\delta_{i j}, \quad\left\{p_{i}, p_{j}\right\}=0
$$

Let us show explicitly that $\vec{J}$ is the generator of spatial rotations so that we can safely define the rank of a tensor by studying its transformation rules under such rotations. Let us prove

$$
\left\{J_{i}, J_{j}\right\}=-\varepsilon_{i j k} J_{k}
$$

Using the tensorial notation for the cross product appearing in the definition of $\vec{J}$, and using the standard properties of a well-define Poisson bracket structure, the brackets in (21) become

$$
\begin{aligned}
\left\{J_{i}, J_{l}\right\}= & \left\{\varepsilon_{i j k} r_{j} p_{k}, \varepsilon_{l m n} r_{m} p_{n}\right\}-\left\{\varepsilon_{i j k} r_{j} p_{k}, \varepsilon_{l m n} r_{m} A_{n}\right\} \\
& -\left\{\varepsilon_{i j k} r_{j} A_{k}, \varepsilon_{l m n} r_{m} p_{n}\right\}+\left\{\varepsilon_{i j k} r_{j} A_{k}, \varepsilon_{l m n} r_{m} A_{n}\right\}+\left\{s_{i}, s_{l}\right\} .
\end{aligned}
$$

Using the basic canonical Poisson bracket structure expressed in (20) and the standard properties of Poisson brackets together with the following identity

$$
\varepsilon_{i j k} \varepsilon_{m l k}=\delta_{i m} \delta_{j l}-\delta_{i l} \delta_{j m}
$$

the first bracket on the right-hand side of (22) becomes

$$
\left\{\varepsilon_{i j k} r_{j} p_{k}, \varepsilon_{l m n} r_{m} p_{n}\right\}=r_{l} p_{i}-r_{i} p_{l}
$$

Similarly, the second, the third, and the fourth brackets on the right-hand side of (22) become

$$
\begin{aligned}
-\left\{\varepsilon_{i j k} r_{j} p_{k}, \varepsilon_{l m n} r_{m} A_{n}\right\} & =\delta_{i l} r_{n} A_{n}-r_{l} A_{i}+\varepsilon_{i j k} \varepsilon_{l m n} r_{m} p_{k}\left\{A_{n}, r_{j}\right\}, \\
-\left\{\varepsilon_{i j k} r_{j} A_{k}, \varepsilon_{l m n} r_{m} p_{n}\right\} & =-\delta_{i l} r_{k} A_{k}+r_{i} A_{l}+\varepsilon_{i j k} \varepsilon_{l m n} r_{j} p_{n}\left\{r_{m}, A_{k}\right\}, \\
\left\{\varepsilon_{i j k} r_{j} A_{k}, \varepsilon_{l m n} r_{m} A_{n}\right\} & =-\varepsilon_{i j k} \varepsilon_{l m n} r_{j} A_{n}\left\{r_{m}, A_{k}\right\}-\varepsilon_{i j k} \varepsilon_{l m n} r_{m} A_{k}\left\{A_{n}, r_{j}\right\} .
\end{aligned}
$$

The last bracket on the right-hand side of (22) is given by (17) Finally, substituting these five brackets in the right-hand side. of (22) and ordering them properly, the Poisson brackets of $\vec{J}$ become

$$
\begin{aligned}
\left\{J_{i}, J_{l}\right\}= & \left(r_{l} p_{i}-r_{i} p_{l}-r_{l} A_{i}+r_{i} A_{l}-\varepsilon_{i l m} s_{m}\right) \\
& +\varepsilon_{i j k} \varepsilon_{l m n}\left[r_{m} p_{k}\left\{A_{n}, r_{j}\right\}-r_{j} p_{n}\left\{r_{m}, A_{k}\right\}\right] \\
& +\varepsilon_{i j k} \varepsilon_{l m n}\left[r_{j} A_{n}\left\{A_{k}, r_{m}\right\}-r_{m} A_{k}\left\{A_{n}, r_{j}\right\}\right] .
\end{aligned}
$$


Because of the full antisymmetry of the Levi-Civita tensor,

$$
\varepsilon_{i j k} \varepsilon_{l m n} r_{m} p_{k}\left\{A_{n}, r_{j}\right\}-\varepsilon_{i j k} \varepsilon_{l m n} r_{j} p_{n}\left\{A_{n}, r_{m}\right\}=\left(\varepsilon_{i j k} \varepsilon_{l m n}-\varepsilon_{i m n} \varepsilon_{l j k}\right) r_{m} p_{k}\left\{A_{n}, r_{j}\right\}=0 .
$$

Therefore, (26) becomes

$$
\begin{aligned}
\left\{J_{i}, J_{l}\right\} & =r_{l} p_{i}-r_{i} p_{l}-r_{l} A_{i}+r_{i} A_{l}-\varepsilon_{i l m} s_{m} \\
& =-\varepsilon_{i l m}\left[\varepsilon_{m n k} r_{n}\left(p_{k}-A_{k}\right)+s_{m}\right] .
\end{aligned}
$$

Using (23), we obtain

$$
-\varepsilon_{i l m} \varepsilon_{m n k} r_{n} p_{k}=\left(r_{l} p_{i}-r_{i} p_{l}\right), \quad \varepsilon_{i l m} \varepsilon_{m n k} r_{n} A_{k}=-\left(r_{l} A_{i}-r_{i} A_{l}\right),
$$

and finally,

$$
\left\{J_{i}, J_{l}\right\}=-\varepsilon_{i l m} J_{m}
$$

At this point, we have all the elements to show the classical inconsistency of the problem. Recall the kinetic momentum vector is defined as

$$
\vec{P} \stackrel{\text { def }}{=} \vec{p}-\frac{e}{c} \vec{A}, \quad \vec{A}=\vec{A}_{\gamma}+\vec{A}^{\text {(Dirac) }} .
$$

Let us assume that there exists a well-defined Poisson bracket structure in the classical theoretical setting in consideration. In particular, let us assume a well-defined classical Poisson bracket structure among the vector fields $\vec{J}, \vec{P}$, and $\vec{r}$, that is,

$$
\left\{J_{i}, J_{j}\right\}=-\varepsilon_{i j k} J_{k}, \quad\left\{J_{i}, r_{j}\right\}=-\varepsilon_{i j k} r_{k}, \quad\left\{J_{i}, P_{j}\right\}=-\varepsilon_{i j k} P_{k} .
$$

Being $\vec{J}$ the generator of rotations, it is required that any arbitrary vector $\vec{v}$ must satisfy the following classical commutation rules:

$$
\left\{J_{i}, v_{j}\right\}=-\varepsilon_{i j k} v_{k} .
$$

Therefore, let us study the transformation properties of the magnetic field under spatial rotations. It must be

$$
\left\{J_{i}, B_{j}\right\}=-\varepsilon_{i j k} B_{k}
$$

In terms of the magnetic field decomposition, (34) is equivalent to

$$
\left\{J_{i}, B_{j}^{\text {(Dirac) }}\right\}=-\varepsilon_{i j k} B_{k}^{(\text {Dirac })}, \quad\left\{J_{i},\left(B_{\gamma}\right)_{j}\right\}=-\varepsilon_{i j k}\left(B_{\gamma}\right)_{k} .
$$

It is quite straightforward to check the validity of the first equation in (35), as a matter of fact,

$$
\begin{aligned}
\left\{J_{i}, B_{j}^{(\text {Dirac })}\right\} & =\left\{J_{i}, \frac{e_{m}}{r^{3}} r_{j}\right\}=\frac{e_{m}}{r^{3}}\left\{J_{i}, r_{j}\right\}+\left\{J_{i}, \frac{e_{m}}{r^{3}}\right\} r_{j} \\
& =-\varepsilon_{i j k} \frac{e_{m}}{r^{3}} r_{k} \equiv-\varepsilon_{i j k} B_{k}^{(\text {Dirac })}
\end{aligned}
$$


Let us consider the validity of (34), where the total magnetic field $\vec{B}$ is given by

$$
B_{j}(r, \theta, \varphi)=\varepsilon_{j l m} \partial_{l} A_{m}(r, \theta, \varphi)+e_{m} f_{j}(\vec{r}) .
$$

By virtue of the Dirac-veto, the magnetic field $B_{j}(r, \theta, \varphi)$ felt by the electric charge reduces to

$$
B_{j}(r, \theta, \varphi)=\varepsilon_{j l m} \partial_{l} A_{m}(r, \theta, \varphi) .
$$

Fixing the constants $c$ and $e$ equal to one for the sake of convenience, let us consider first the Poisson brackets of the kinetic momentum vector components. Using (20), the standard properties of Poisson brackets together with (23) and (38), we obtain

$$
\left\{P_{i}, P_{j}\right\}=-\varepsilon_{i j k} B_{k}
$$

Multiplying both sides of (39) by $\varepsilon_{i j n}$, we obtain

$$
\varepsilon_{i j n}\left\{P_{i}, P_{j}\right\}=-\varepsilon_{i j n} \varepsilon_{i j k} B_{k}=-2 \delta_{n k} B_{k}=-2 B_{n}
$$

and therefore

$$
B_{k}=-\frac{1}{2} \varepsilon_{i j k}\left\{P_{i}, P_{j}\right\}
$$

Therefore, substituting $B_{k}$ of (41) into (34), we obtain

$$
\left\{J_{i}, B_{j}\right\}=-\frac{1}{2} \varepsilon_{l m j}\left\{J_{i},\left\{P_{l}, P_{m}\right\}\right\} .
$$

The double commutator in (42) cannot be calculated in a direct way. However, because we are assuming the existence of a well-defined Poisson bracket structure among the vectors $\vec{J}, \vec{B}$, and $\vec{r}$, this double commutator can be evaluated by using the following Jacobi identity

$$
\left\{J_{i},\left\{P_{l}, P_{m}\right\}\right\}+\left\{P_{m},\left\{J_{i}, P_{l}\right\}\right\}+\left\{P_{l},\left\{P_{m}, J_{i}\right\}\right\}=0 .
$$

Thus, using the fact that $\vec{J}$ is the generator of rotations, that $\vec{P}$ transforms as a vector quantity under rotations, and using (23), we obtain

$$
\left\{J_{i},\left\{P_{l}, P_{m}\right\}\right\}=-\delta_{i l} B_{m}+\delta_{i m} B_{l}
$$

Substituting (41) into (44), we obtain

$$
\left\{J_{i}, B_{j}\right\}=-\varepsilon_{i j m} B_{m}
$$

Therefore, we have shown that in a pure classical theoretical framework given by the Poisson brackets formalism, the commutation rule between the generator of spatial rotations and the total magnetic field is expressed in (45). Our last step is to calculate the Poisson brackets between $\vec{J}$ and the magnetic field $\vec{B}_{\gamma}$. Using (5), standard Poisson brackets properties, and the fact that $\vec{J}$ is the generator of rotations, these brackets become

$$
\left\{J_{i},\left(\vec{B}_{\gamma}\right)_{j}\right\}_{\text {Poisson }}=-\varepsilon_{i j k}\left(B_{\gamma}\right)_{k}+\left\{J_{i}, b_{\gamma}^{(1)}\right\} r_{j}+\left\{J_{i}, b_{\gamma}^{(2)}\right\} n_{j}
$$


In order to have proper Poisson brackets, for each vectors $\widehat{n}$ and $\vec{r}$, the following relation must hold:

$$
\left\{J_{i}, b_{\gamma}^{(1)}\right\} r_{j}+\left\{J_{i}, b_{\gamma}^{(2)}\right\} n_{j}=0
$$

Observe that the second Poisson bracket in the right-hand side of (46) contains a term quadratic in $n_{k}$,

$$
\begin{aligned}
\left\{J_{i}, b_{\gamma}^{(2)}\right\} n_{j} & =\left(\partial_{p_{k}} J_{i}\right)\left(\partial_{r_{k}} b_{\gamma}^{(2)}\right) n_{j} \\
& =\left(\partial_{p_{k}} J_{i}\right)\left[\partial_{r} b_{\gamma}^{(2)} \frac{r_{k}}{r}+\partial_{(\vec{r} \cdot \hat{n})} b_{r}^{(2)} n_{k}\right] n_{j} \\
& =\frac{1}{r} \partial_{p_{k}} J_{i} \partial_{r} b_{\gamma}^{(2)} r_{k} n_{j}+\partial_{p_{k}} J_{i} \partial_{(\vec{r} \cdot \hat{n})} b_{r}^{(2)} n_{k} n_{j}
\end{aligned}
$$

Since the proper Poisson brackets should be linear in $n_{k}$, we require

$$
\partial_{(\vec{r} \cdot \widehat{n})} b_{r}^{(2)}=0
$$

There is no way to cancel out this term in (46), then it must be

$$
b_{\gamma}^{(2)}=0
$$

We now consider the first Poisson bracket on the right-hand side of (46). Because of the antisymmetry in the indices $i$ and $j$ of the term $\varepsilon_{i j k}\left(B_{\gamma}\right)_{k}$, it must be

$$
\left\{J_{i}, b_{\gamma}^{(1)}\right\} r_{j}+\left\{J_{j}, b_{\gamma}^{(1)}\right\} r_{i}=0
$$

that is,

$$
\left\{J_{i}, b_{\gamma}^{(1)}\right\} r_{i}=0
$$

Explicitly, (52) becomes

$$
\begin{aligned}
0 & =\left(\partial_{p_{k}} J_{i}\right)\left(\partial_{r_{k}} b_{\gamma}^{(1)}\right) r_{i} \\
& =\left(\partial_{p_{k}} J_{i}\right)\left[\partial_{r} b_{\gamma}^{(1)} \frac{r_{k}}{r}+\partial_{(\vec{r} \cdot \hat{n})} b_{\gamma}^{(1)} n_{k}\right] r_{i} \\
& =\frac{1}{r}\left(\partial_{p_{k}} J_{i}\right)\left(\partial_{r} b_{\gamma}^{(1)}\right) r_{k} r_{i}+\left(\partial_{p_{k}} J_{i}\right)\left(\partial_{(\vec{r} \cdot \hat{n})} b_{\gamma}^{(1)}\right) n_{k} r_{i} .
\end{aligned}
$$

We neglect the quadratic term in $r_{k}$ in (53) since this term has no analog in the proper Poisson brackets. Then, we have

$$
\partial_{(\vec{r} \cdot \widehat{n})} b_{\gamma}^{(1)}=0
$$

Recalling that

$$
\widehat{n}=-\widehat{z}=-\{\cos (\theta) \widehat{r}-\sin (\theta) \widehat{\theta}\}=-\cos (\theta) \widehat{r}+\sin (\theta) \widehat{\theta}
$$


then

$$
\widehat{n} \cdot \widehat{r}=-\cos (\theta)=\theta \text {-dependent. }
$$

Therefore, (54) is satisfied by an arbitrary scalar function $b_{\gamma}(r)$. As a consequence, the magnetic field $\vec{B}_{\gamma}$ is not $\theta$-dependent (in a more general situation in which $\widehat{n}$ is not along the $z$-axis, we would conclude that the magnetic field is not $(\theta, \varphi)$-dependent). $\vec{B}_{\gamma}$ must be a spherically symmetric field whose general expression is the following:

$$
\vec{B}_{\gamma}(\vec{r})=B_{\gamma}(r) \widehat{r}
$$

In conclusion, in order to have a well-defined classical Poisson bracket structure in the problem under investigation, one must deal with diffuse magnetic field solutions exhibiting spherical symmetry. However, those very same solutions are not compatible with massive classical electrodynamics with magnetic monopoles. This result means that it is not possible to formulate a consistent nonrelativistic classical theory describing the finite-range electromagnetic interaction between a point-like electric charge and a fixed Dirac monopole without a visible string. In other words, there is no way to construct a consistent Lie algebra in our classical framework and this leads to the conclusion that there is no angular momentum to be quantized in order to give the Dirac quantization rule. This fact points out that the string attached to the monopole is visible and there is no way to make it invisible when considering finite-range electromagnetic interactions in a pure classical framework. The Dirac string must assume dynamical significance if the photon has a nonvanishing mass, and its dynamical evolution may play a significant role in a quantum description of the Dirac theory. In conclusion, we have shown that it is not possible to construct a nonrelativistic classical theory of true Dirac monopoles (invisible string, "monopole without a string") and massive photons unless the string attached to the monopole is treated as an independent dynamical quantity. An important feature of our approach is that we do not use any kind of semiclassical approximation or limiting procedure for $\hbar \rightarrow 0$.

\section{Appendices}

\section{A. The generator of spatial rotations}

We show that $\vec{J}$ is the generator of spatial rotations, that is,

$$
\left\{J_{i}, J_{j}\right\}=-\varepsilon_{i j k} J_{k}
$$

Notice that

$$
\begin{aligned}
\left\{J_{i}, J_{l}\right\}= & \left\{\varepsilon_{i j k} r_{j}\left(p_{k}-A_{k}\right)+s_{i}, \varepsilon_{l m n} r_{m}\left(p_{n}-A_{n}\right)+s_{l}\right\} \\
= & \left\{\varepsilon_{i j k} r_{j} p_{k}-\varepsilon_{i j k} r_{j} A_{k}+s_{i}, \varepsilon_{l m n} r_{m} p_{n}-\varepsilon_{l m n} r_{m} A_{n}+s_{l}\right\} \\
= & \left\{\varepsilon_{i j k} r_{j} p_{k}, \varepsilon_{l m n} r_{m} p_{n}\right\}-\left\{\varepsilon_{i j k} r_{j} p_{k}, \varepsilon_{l m n} r_{m} A_{n}\right\}-\left\{\varepsilon_{i j k} r_{j} A_{k}, \varepsilon_{l m n} r_{m} p_{n}\right\} \\
& +\left\{\varepsilon_{i j k} r_{j} A_{k}, \varepsilon_{l m n} r_{m} A_{n}\right\}+\left\{s_{i}, s_{l}\right\} .
\end{aligned}
$$


Therefore, there are five Poisson brackets to be calculated. Consider the first one

$$
\begin{aligned}
\left\{\varepsilon_{i j k} r_{j} p_{k}, \varepsilon_{l m n} r_{m} p_{n}\right\}= & \varepsilon_{i j k} \varepsilon_{l m n}\left\{r_{j} p_{k}, r_{m} p_{n}\right\} \\
= & \varepsilon_{i j k} \varepsilon_{l m n}\left[r_{j}\left\{p_{k}, r_{m} p_{n}\right\}+\left\{r_{j}, r_{m} p_{n}\right\} p_{k}\right] \\
= & \varepsilon_{i j k} \varepsilon_{l m n}\left[-r_{j}\left\{r_{m} p_{n}, p_{k}\right\}-\left\{r_{m} p_{n}, r_{j}\right\} p_{k}\right] \\
= & \varepsilon_{i j k} \varepsilon_{l m n}\left[-r_{j}\left(r_{m}\left\{p_{n}, p_{k}\right\}+\left\{r_{m}, p_{k}\right\} p_{n}\right)\right] \\
& +\varepsilon_{i j k} \varepsilon_{l m n}\left[-\left(r_{m}\left\{p_{n}, r_{j}\right\}+\left\{r_{m}, r_{j}\right\} p_{n}\right) p_{k}\right] \\
= & \varepsilon_{i j k} \varepsilon_{l m n}\left[\delta_{m k} r_{j} p_{n}-\delta_{n j} r_{m} p_{k}\right]=\varepsilon_{i j k} \varepsilon_{l m n} \delta_{m k} r_{j} p_{n}-\varepsilon_{i j k} \varepsilon_{l m n} \delta_{n j} r_{m} p_{k} \\
= & \varepsilon_{i j k} \varepsilon_{l k n} r_{j} p_{n}-\varepsilon_{i n k} \varepsilon_{l m n} r_{m} p_{k}=-\varepsilon_{i j k} \varepsilon_{l n k} r_{j} p_{n}+\varepsilon_{i k n} \varepsilon_{l m n} r_{m} p_{k} \\
= & -\left(\delta_{i l} \delta_{j n}-\delta_{i n} \delta_{j l}\right) r_{j} p_{n}+\left(\delta_{i l} \delta_{k m}-\delta_{i m} \delta_{l k}\right) r_{m} p_{k} \\
= & -\delta_{i l} \delta_{j n} r_{j} p_{n}+\delta_{i n} \delta_{j l} r_{j} p_{n}+\delta_{i l} \delta_{k m} r_{m} p_{k}-\delta_{i m} \delta_{l k} r_{m} p_{k} \\
= & -\delta_{i l} r_{n} p_{n}+r_{l} p_{i}+\delta_{i l} r_{k} p_{k}-r_{i} p_{l}=r_{l} p_{i}-r_{i} p_{l},
\end{aligned}
$$

thus

$$
\left\{\varepsilon_{i j k} r_{j} p_{k}, \varepsilon_{l m n} r_{m} p_{n}\right\}=r_{l} p_{i}-r_{i} p_{l}
$$

Consider the second bracket

$$
\begin{aligned}
-\left\{\varepsilon_{i j k} r_{j} p_{k}, \varepsilon_{l m n} r_{m} A_{n}\right\}= & -\varepsilon_{i j k} \varepsilon_{l m n}\left\{r_{j} p_{k}, r_{m} A_{n}\right\} \\
= & -\varepsilon_{i j k} \varepsilon_{l m n}\left[r_{j}\left\{p_{k}, r_{m} A_{n}\right\}+\left\{r_{j}, r_{m} A_{n}\right\} p_{k}\right] \\
= & -\varepsilon_{i j k} \varepsilon_{l m n}\left[-r_{j}\left\{r_{m} A_{n}, p_{k}\right\}-\left\{r_{m} A_{n}, r_{j}\right\} p_{k}\right] \\
= & -\varepsilon_{i j k} \varepsilon_{l m n}\left[-r_{j} r_{m}\left\{A_{n}, p_{k}\right\}-r_{j}\left\{r_{m}, p_{k}\right\} A_{n}\right] \\
& -\varepsilon_{i j k} \varepsilon_{l m n}\left[-r_{m}\left\{A_{n}, r_{j}\right\} p_{k}-\left\{r_{m}, r_{j}\right\} A_{n} p_{k}\right] \\
= & -\varepsilon_{i j k} \varepsilon_{l m n}\left[\delta_{m k} r_{j} A_{n}-r_{m} p_{k}\left\{A_{n}, r_{j}\right\}\right] \\
= & -\varepsilon_{i j k} \varepsilon_{l k n} r_{j} A_{n}+\varepsilon_{i j k} \varepsilon_{l m n} r_{m} p_{k}\left\{A_{n}, r_{j}\right\} \\
= & \varepsilon_{i j k} \varepsilon_{l, n, k} r_{j} A_{n}+\varepsilon_{i j k} \varepsilon_{l m n} r_{m} p_{k}\left\{A_{n}, r_{j}\right\} \\
= & \left(\delta_{i l} \delta_{j n}-\delta_{i n} \delta_{j l}\right) r_{j} A_{n}+\varepsilon_{i j k} \varepsilon_{l m n} r_{m} p_{k}\left\{A_{n}, r_{j}\right\} \\
= & \delta_{i l} \delta_{j n} r_{j} A_{n}-\delta_{i n} \delta_{j l} r_{j} A_{n}+\varepsilon_{i j k} \varepsilon_{l m n} r_{m} p_{k}\left\{A_{n}, r_{j}\right\} \\
= & \delta_{i l} r_{n} A_{n}-r_{l} A_{i}+\varepsilon_{i j k} \varepsilon_{l m n} r_{m} p_{k}\left\{A_{n}, r_{j}\right\},
\end{aligned}
$$

thus

$$
-\left\{\varepsilon_{i j k} r_{j} p_{k}, \varepsilon_{l m n} r_{m} A_{n}\right\}=\delta_{i l} r_{n} A_{n}-r_{l} A_{i}+\varepsilon_{i j k} \varepsilon_{l m n} r_{m} p_{k}\left\{A_{n}, r_{j}\right\} .
$$

Using the standard canonical algebra, the third bracket becomes

$$
-\left\{\varepsilon_{i j k} r_{j} A_{k}, \varepsilon_{l m n} r_{m} p_{n}\right\}=-\delta_{i l} r_{k} A_{k}+r_{i} A_{l}+\varepsilon_{i j k} \varepsilon_{l m n} r_{j} p_{n}\left\{r_{m}, A_{k}\right\} .
$$

For the fourth bracket, we obtain

$$
\begin{aligned}
\left\{\varepsilon_{i j k} r_{j} A_{k}, \varepsilon_{l m n} r_{m} A_{n}\right\} & =\varepsilon_{i j k} \varepsilon_{l m n}\left\{r_{j} A_{k}, r_{m} A_{n}\right\} \\
& =\varepsilon_{i j k} \varepsilon_{l m n}\left[r_{j}\left\{A_{k}, r_{m} A_{n}\right\}+\left\{r_{j}, r_{m} A_{n}\right\} A_{k}\right] \\
& =\varepsilon_{i j k} \varepsilon_{l m n}\left[-r_{j}\left\{r_{m} A_{n}, A_{k}\right\}-\left\{r_{m} A_{n}, r_{j}\right\} A_{k}\right] \\
& =\varepsilon_{i j k} \varepsilon_{l m n}\left[-r_{j}\left\{r_{m}, A_{k}\right\} A_{n}-r_{m}\left\{A_{n}, r_{j}\right\} A_{k}\right] \\
& =-\varepsilon_{i j k} \varepsilon_{l m n} r_{j} A_{n}\left\{r_{m}, A_{k}\right\}-\varepsilon_{i j k} \varepsilon_{l m n} r_{m} A_{k}\left\{A_{n}, r_{j}\right\} .
\end{aligned}
$$


For the last bracket, let us remind that the vector $s$ is such the Poisson brackets of its components satisfy (17). In conclusion, using (A.4), (A.6), (A.7), (A.8), and using the commutation rules of the classical spin, (A.2) becomes

$$
\begin{aligned}
\left\{J_{i}, J_{l}\right\}= & r_{l} p_{i}-r_{i} p_{l}+\delta_{i l} r_{n} A_{n}-r_{l} A_{i}+\varepsilon_{i j k} \varepsilon_{l m n} r_{m} p_{k}\left\{A_{n}, r_{j}\right\}-\delta_{i l} r_{k} A_{k} \\
& +r_{i} A_{l}+\varepsilon_{i j k} \varepsilon_{l m n} r_{j} p_{n}\left\{r_{m}, A_{k}\right\}-\varepsilon_{i j k} \varepsilon_{l m n} r_{j} A_{n}\left\{r_{m}, A_{k}\right\} \\
& -\varepsilon_{i j k} \varepsilon_{l m n} r_{m} A_{k}\left\{A_{n}, r_{j}\right\}-\varepsilon_{i l m} s_{m} \\
= & \left(r_{l} p_{i}-r_{i} p_{l}-r_{l} A_{i}+r_{i} A_{l}-\varepsilon_{i l m} s_{m}\right) \\
& +\left(\varepsilon_{i j k} \varepsilon_{l m n}\left[r_{m} p_{k}\left\{A_{n}, r_{j}\right\}-r_{j} p_{n}\left\{r_{m}, A_{k}\right\}+r_{j} A_{n}\left\{A_{k}, r_{m}\right\}-r_{m} A_{k}\left\{A_{n}, r_{j}\right\}\right]\right) .
\end{aligned}
$$

Notice that

$$
\varepsilon_{i j k} \varepsilon_{l m n} r_{m} p_{k}\left\{A_{n}, r_{j}\right\}-\varepsilon_{i j k} \varepsilon_{l m n} r_{j} p_{n}\left\{A_{n}, r_{m}\right\}=\left(\varepsilon_{i j k} \varepsilon_{l m n}-\varepsilon_{i m n} \varepsilon_{l j k}\right) r_{m} p_{k}\left\{A_{n}, r_{j}\right\}=0 .
$$

If $i=l$, then

$$
\varepsilon_{i j k} \varepsilon_{l m n}-\varepsilon_{i m n} \varepsilon_{l j k}=\varepsilon_{i j k} \varepsilon_{i m n}-\varepsilon_{i m n} \varepsilon_{i j k} \equiv 0 .
$$

If $i \neq l$, let us say $i=1$ and $l=2$, then

$$
\varepsilon_{i j k} \varepsilon_{l m n}-\varepsilon_{i m n} \varepsilon_{l j k}=\varepsilon_{1 j k} \varepsilon_{2 m n}-\varepsilon_{1 m n} \varepsilon_{2 j k} .
$$

Therefore, the possible nonvanishing pieces are

$$
\varepsilon_{123} \varepsilon_{213}-\varepsilon_{213} \varepsilon_{123} \equiv 0, \quad \varepsilon_{132} \varepsilon_{231}-\varepsilon_{231} \varepsilon_{132} \equiv 0, \quad \varepsilon_{132} \varepsilon_{213}-\varepsilon_{231} \varepsilon_{123} \equiv 0, \ldots
$$

Therefore, (A.9) becomes

$$
\begin{aligned}
\left\{J_{i}, J_{l}\right\} & =\left(r_{l} p_{i}-r_{i} p_{l}-r_{l} A_{i}+r_{i} A_{l}-\varepsilon_{i l m} s_{m}\right) \\
& =-\varepsilon_{i l m}\left[\varepsilon_{m n k} r_{n}\left(p_{k}-A_{k}\right)+s_{m}\right] \\
& =-\varepsilon_{i l m} J_{m} .
\end{aligned}
$$

Indeed,

$$
\begin{aligned}
-\varepsilon_{i l m} \varepsilon_{m n k} r_{n} p_{k} & =-\varepsilon_{i l m} \varepsilon_{k m n} r_{n} p_{k}=-\varepsilon_{i l m} \varepsilon_{n k m} r_{n} p_{k} \\
& =\left(\delta_{i n} \delta_{l k}-\delta_{i k} \delta_{l, n}\right) r_{n} p_{k}=-\delta_{i n} \delta_{l k} r_{n} p_{k}+\delta_{i k} \delta_{l, n} r_{n} p_{k} \\
& =-r_{i} p_{l}+r_{l} p_{i}=\left(r_{l} p_{i}-r_{i} p_{l}\right), \\
\varepsilon_{i l m} \varepsilon_{m n k} r_{n} A_{k} & =r_{i} A_{l}+r_{l} A_{i}=-\left(r_{l} A_{i}-r_{i} A_{l}\right) .
\end{aligned}
$$

This concludes our proof. 


\section{B. The Jacobi identity}

Consider the kinetic momentum vector

$$
\vec{P} \stackrel{\text { def }}{=} \vec{p}-\frac{e}{c} \vec{A}, \quad \vec{A}=\vec{A}_{\gamma}+\vec{A}^{\text {(Dirac) }} .
$$

Consider the Poisson bracket of the kinetic momentum vector components

$$
\begin{aligned}
\left\{P_{i}, P_{j}\right\} & =\left\{p_{i}-A_{i}, p_{j}-A_{j}\right\} \\
& =\left\{p_{i}, p_{j}\right\}-\left\{p_{i}, A_{j}\right\}-\left\{A_{i}, p_{j}\right\}+\left\{A_{i}, A_{j}\right\}=\left\{A_{j}, p_{i}\right\}-\left\{A_{i}, p_{j}\right\} \\
& =\left\{A_{j}, p_{i}\right\}-\left\{A_{i}, p_{j}\right\}=-\partial i A_{j}+\partial_{j} A_{i}=-\left(\partial i A_{j}-\partial_{j} A_{i}\right) \\
& =-\varepsilon_{i j k} B_{k},
\end{aligned}
$$

where

$$
B_{j}=\varepsilon_{j l m} \partial_{l} A_{m} .
$$

Using the fact that $\left\{J_{i}, B_{j}\right\}=-\varepsilon_{i j k} B_{k}$ and the identity $\varepsilon_{i j k} \varepsilon_{m l k}=\delta_{i l} \delta_{j m}-\delta_{i m} \delta_{j l}$, it follows that

$$
\begin{aligned}
\varepsilon_{i j k} B_{k} & =\varepsilon_{i j k} \varepsilon_{k l m} \partial_{l} A_{m}=\varepsilon_{i j k} \varepsilon_{m k l} \partial_{l} A_{m}=-\varepsilon_{i j k} \varepsilon_{m l k} \partial_{l} A_{m} \\
& =-\left(\delta_{i m} \delta_{j l}-\delta_{i l} \delta_{j m}\right) \partial_{l} A_{m}=-\delta_{i m} \delta_{j l} \partial_{l} A_{m}+\delta_{i l} \delta_{j m} \partial_{l} A_{m} \\
& =-\delta_{i m} \partial_{j} A_{m}+\delta_{i l} \partial_{l} A_{j}=\partial_{i} A_{j}-\partial_{j} A_{i} .
\end{aligned}
$$

Using (B.2), we obtain

$$
\varepsilon_{i j n}\left\{P_{i}, P_{j}\right\}=-\varepsilon_{i j n} \varepsilon_{i j k} B_{k}=-2 \delta_{n k} B_{k}=-2 B_{n} .
$$

Thus,

$$
B_{k}=-\frac{1}{2} \varepsilon_{i j k}\left\{P_{i}, P_{j}\right\} .
$$

Finally, let us focus on the following Poisson bracket:

$$
\left\{J_{i}, B_{j}\right\}=\left\{J_{i},-\frac{1}{2} \varepsilon_{l m j}\left\{P_{l}, P_{m}\right\}\right\}=-\frac{1}{2} \varepsilon_{l m j}\left\{J_{i},\left\{P_{l}, P_{m}\right\}\right\} .
$$

Using the Jacobi identity

$$
\left\{J_{i},\left\{P_{l}, P_{m}\right\}\right\}+\left\{P_{m},\left\{J_{i}, P_{l}\right\}\right\}+\left\{P_{l},\left\{P_{m}, J_{i}\right\}\right\}=0,
$$

we obtain

$$
\begin{aligned}
\left\{J_{i,}\left\{P_{l}, P_{m}\right\}\right\} & =-\left\{P_{m,}\left\{J_{i}, P_{l}\right\}\right\}-\left\{P_{l},\left\{P_{m}, J_{i}\right\}\right\} \\
& =\left\{P_{l},\left\{J_{i}, P_{m}\right\}\right\}-\left\{P_{m,}\left\{J_{i}, P_{l}\right\}\right\} \\
& =\left\{P_{l},-\varepsilon_{i m k} P_{k}\right\}-\left\{P_{m},-\varepsilon_{i l k} P_{k}\right\} \\
& =-\varepsilon_{i m k}\left\{P_{l}, P_{k}\right\}+\varepsilon_{i l k}\left\{P_{m}, P_{k}\right\} \\
& =-\varepsilon_{i m k}\left(-\varepsilon_{l k q} B_{q}\right)+\varepsilon_{i l k}\left(-\varepsilon_{m k q} B_{q}\right) \\
& =\varepsilon_{i m k} \varepsilon_{l k q} B_{q}-\varepsilon_{i l k} \varepsilon_{m k q} B_{q} \\
& =-\varepsilon_{i m k} \varepsilon_{l q k} B_{q}+\varepsilon_{i l k} \varepsilon_{m q k} B_{q} \\
& =-\left(\delta_{i l} \delta_{m q}-\delta_{i q} \delta_{m l}\right) B_{q}+\left(\delta_{i m} \delta_{l q}-\delta_{i q} \delta_{l m}\right) B_{q} \\
& =-\delta_{i l} \delta_{m q} B_{q}+\delta_{i q} \delta_{m l} B_{q}+\delta_{i m} \delta_{l q} B_{q}-\delta_{i q} \delta_{l m} B_{q} \\
& =-\delta_{i l} B_{m}+\delta_{m l} B_{i}+\delta_{i m} B_{l}-\delta_{l m} B_{i}=-\delta_{i l} B_{m}+\delta_{i m} B_{l} .
\end{aligned}
$$


Then, using (B.6) and (B.9), we obtain

$$
\begin{aligned}
\left\{J_{i}, B_{j}\right\} & =-\frac{1}{2} \varepsilon_{l m j}\left(-\delta_{i l} B_{m}+\delta_{i m} B_{l}\right)=\frac{1}{2} \varepsilon_{l m j} \delta_{i l} B_{m}-\frac{1}{2} \varepsilon_{l m j} \delta_{i m} B_{l} \\
& =\frac{1}{2} \varepsilon_{i m j} B_{m}-\frac{1}{2} \varepsilon_{l i j} B_{l}=-\frac{1}{2} \varepsilon_{i j m} B_{m}-\frac{1}{2} \varepsilon_{m i j} B_{m} \\
& =-\frac{1}{2} \varepsilon_{i j m} B_{m}-\frac{1}{2} \varepsilon_{i j m} B_{m}=-\varepsilon_{i j m} B_{m} .
\end{aligned}
$$

We have shown that in a pure classical theoretical framework given by the Poisson brackets formalism, the commutation rule between the generator of spatial rotations and the total magnetic field is

$$
\left\{J_{i}, B_{j}\right\}=i \varepsilon_{i j k} B_{k}
$$

\section{Acknowledgments}

The authors are grateful to A. Caticha and J. Kimball for their useful comments.

\section{References}

[1] P. A. M. Dirac, "Quantized singularities in the electromagnetic field," Proceedings of the Royal Society, vol. A133, pp. 60-72, 1931.

[2] P. A. M. Dirac, "The theory of magnetic poles," Physical Review, vol. 74, no. 7, pp. 817-830, 1948.

[3] R. Jackiw, "Three-cocycle in mathematics and physics," Physical Review Letters, vol. 54, no. 3, pp. 159$162,1985$.

[4] C. Nash, Differential Topology and Quantum Field Theory, Academic Press, New York, NY, USA, 1991.

[5] T. T. Wu and C. N. Yang, "Concept of nonintegrable phase factors and global formulation of gauge fields," Physical Review D, vol. 12, no. 12, pp. 3845-3857, 1975.

[6] T. T. Wu and C. N. Yang, "Dirac monopole without strings: monopole harmonics," Nuclear Physics B, vol. B107, no. 3, pp. 365-380, 1976.

[7] A. Yu Ignatiev and G. C. Joshi, "Massive electrodynamics and the magnetic monopoles," Physical Review D, vol. 53, no. 2, pp. 984-992, 1996.

[8] A. P. Balachandran, R. Ramachandran, J. Schechter, et al., "Hamiltonian formulation of monopole theories with strings," Physical Review D, vol. 13, no. 2, pp. 354-360, 1975.

[9] A. I. Nesterov and F. Aceves de la Cruz, "Towards a new quantization of Dirac's monopole," Revista Mexicana de Física, vol. 49, suppl. 2, pp. 134-136, 2003.

[10] J. Schwinger, "Magnetic charge and quantum field theory," Physical Review, vol. 144, no. 4, pp. 10871093, 1966.

[11] J. Schwinger, "Electric and magnetic charge renormalization. I," Physical Review, vol. 151, no. 4, pp. 1048-1054, 1966.

[12] J. Schwinger, "Electric and magnetic charge renormalization. II," Physical Review, vol. 151, no. 4, pp. 1055-1057, 1966.

[13] D. Zwanziger, "Local Lagrangian quantum field theory of electric and magnetic charges," Physical Review D, vol. 3, no. 4, pp. 880-891, 1971.

[14] Israelit M., "Massive electrodynamics and magnetic monopoles," General Relativity and Gravitation, vol. 29, no. 11, pp. 1411-1424, 1997.

[15] Israelit M., "Magnetic monopoles and massive photons in a Weyl-type electrodynamics," General Relativity and Gravitation, vol. 29, no. 12, pp. 1597-1614, 1997.

[16] K. A. Milton, "Theoretical and experimental status of magnetic monopoles," Reports on Progress in Physics, vol. 69, no. 6, pp. 1637-1711, 2006. 
[17] R. A. Brandtand and J. R. Primack, "Avoiding "Dirac's veto" in monopole theory," Physical Review D, vol. 15, no. 6, pp. 1798-1802, 1977.

[18] C. Cafaro and S. A. Ali, "The spacetime algebra approach to massive classical electrodynamics with magnetic monopoles," Advances in Applied Clifford Algebras, vol. 17, no. 1, pp. 23-36, 2007.

[19] C. Cafaro, "Finite-range electromagnetic interaction and magnetic charges: spacetime algebra or algebra of physical space?" Advances in Applied Clifford Algebras, vol. 17, no. 4, pp. 617-634, 2007.

[20] E. Kyriakopoulos, "Equations of a massive electrodynamics with magnetic charges," Physical Review D, vol. 16, no. 2, pp. 517-519, 1977.

[21] A. S. Goldhaber, "Role of spin in the monopole problem," Physical Review, vol. D140, no. 5B, pp. B1407B1414, 1965.

[22] W. A. Moura-Melo, N. Panza, J. A. Helayël-Neto, et al., "On massive vector bosons and abelian magnetic monopoles in $D=(3+1)$ : a possible way to quantise topological mass parameter," International Journal of Modern Physics A, vol. 14, no. 25, pp. 3949-3961, 1999.

[23] A. Berard, Y. Grandati, and H. Mohrbach, "Dirac monopole with Feynman brackets," Physics Letters A, vol. 254, no. 3-4, pp. 133-136, 1999. 

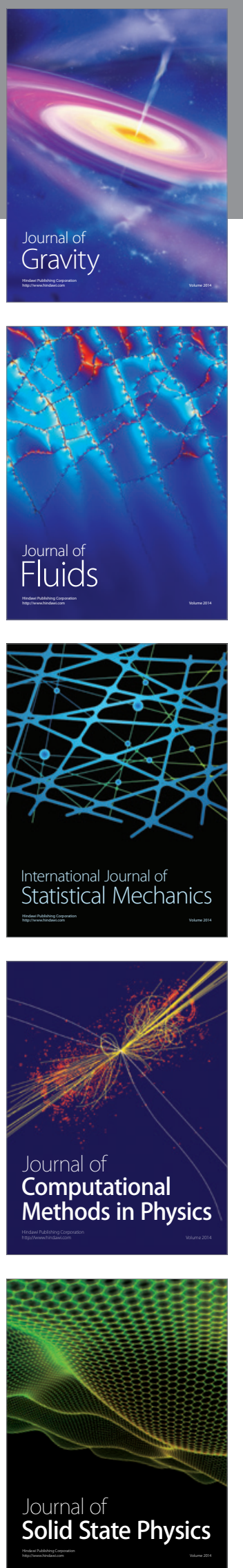

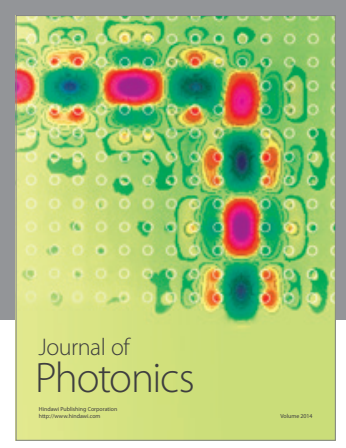

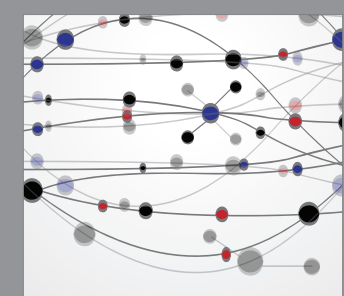

The Scientific World Journal
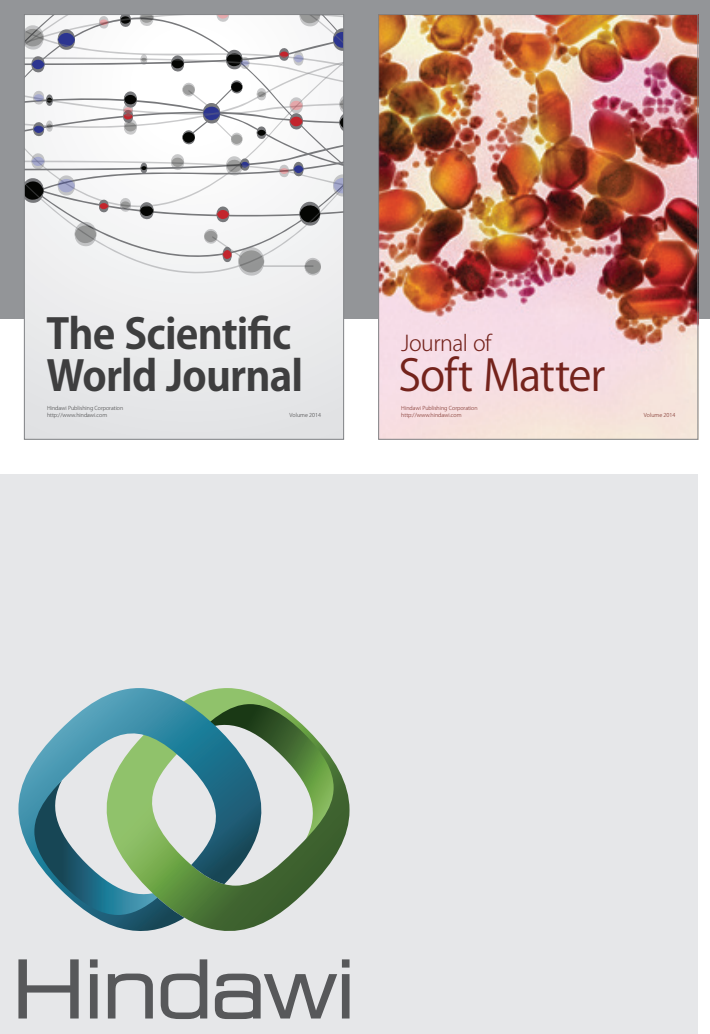

Submit your manuscripts at

http://www.hindawi.com
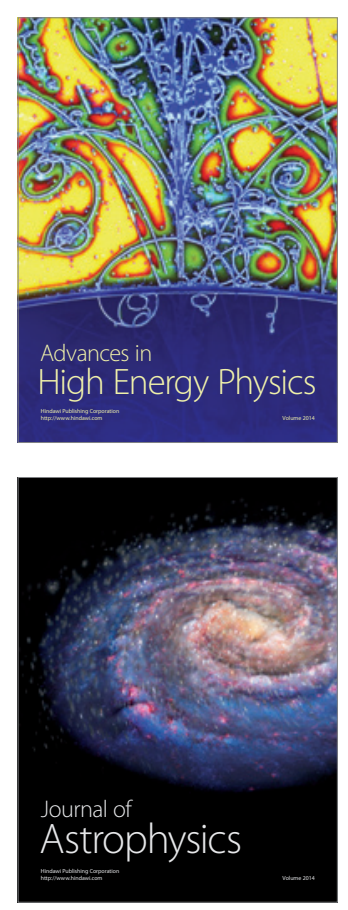
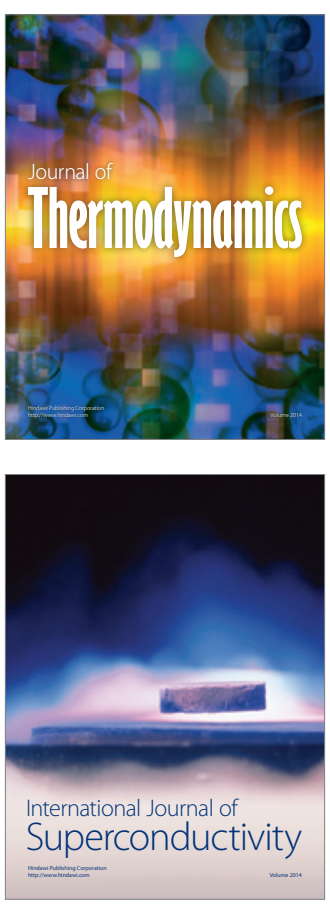
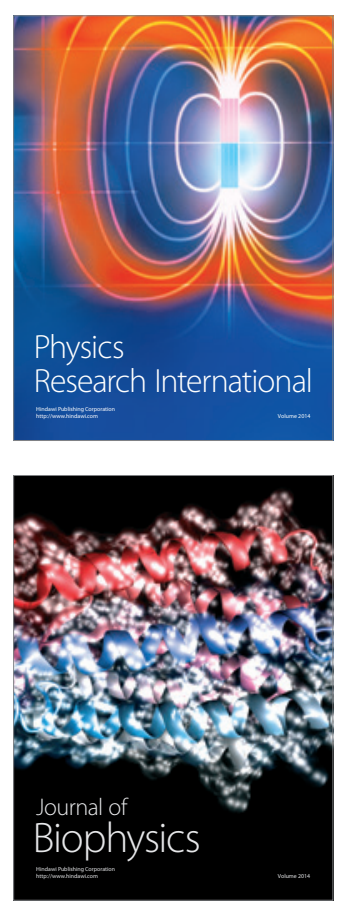
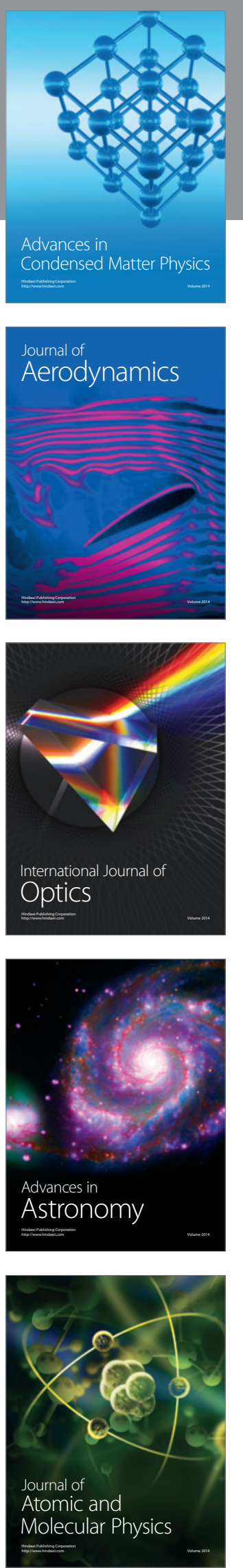